\title{
How Artificial Intelligence May Be Applied in Real World Situations
}

\author{
Zbigniew Michalewicz \\ University of Adelaide, Adelaide, Australia \\ zbyszek@cs.adelaide.edu.au
}

\begin{abstract}
In the modern information era, managers must recognize the competitive opportunities represented by decision-support tools. New family of such systems, based on recent advances in Artificial Intelligence, combine prediction and optimization techniques to assist decision makers in complex, rapidly changing environments. These systems address the fundamental questions: What is likely to happen in the future? and what is the best course of action? These modern AI systems include elements of data mining, predictive modelling, forecasting, optimization, and adaptability and aim at providing significant cost savings and revenue increases for businesses. The talk introduces the concepts behind construction of such systems and indicates the current challenging research issues. Several real-world examples will be shown and discussed.
\end{abstract}

